\title{
Heat shock cognate protein 70 promotes the differentiation of C2C12 myoblast and targets Yin Yang 1
}

\author{
Lei Chen ${ }^{1 \#}$, Tao Guo ${ }^{2 \#}$, Yan Yu ${ }^{1}$, Yeqing Sun ${ }^{1}$, Guangrong Yu ${ }^{1}$, Liming Cheng $^{1,3}$ \\ ${ }^{1}$ Department of Orthopedics, Tongji Hospital, Tongji University School of Medicine, Shanghai 200065, China; ${ }^{2}$ Department of Orthopedics, \\ Guizhou Provincial People's Hospital, Guiyang 550002, China; ${ }^{3}$ Key Laboratory of Spine and Spinal Cord Injury Repair and Regeneration, Ministry \\ of Education of the People's Republic of China, Tongji Hospital Affiliated to Tongji University School of Medicine, Shanghai 200065, China \\ Contributions: (I) Conception and design: L Cheng, L Chen, T Guo, G Yu; (II) Administrative support: Y Yu, Y Sun; (III) Provision of study materials \\ or patients: L Chen, T Guo, Y Yu, Y Sun; (IV) Collection and assembly of data: L Chen, T Guo, Y Yu, Y Sun; (V) Data analysis and interpretation: \\ All authors; (VI) Manuscript writing: All authors; (VII) Final approval of manuscript: All authors. \\ \#These authors contributed equally to this work. \\ Correspondence to: Liming Cheng; Guangrong Yu. Department of Orthopedics, Tongji Hospital, Tongji University School of Medicine, 389 Xincun \\ Road, Shanghai 200065, China. Email: limingcheng@tongji.edu.cn; Yuguangrong@hotmail.com.
}

\begin{abstract}
Background: Heat shock cognate protein 70 (HSC70) is a constitutively expressed molecular chaperone protein which can maintain the structure and function of the protein. HSC70 is engaged in a variety of physiological processes, yet its role during skeletal muscle differentiation is still unclear.

Methods: C2C12 cells were obtained and cultured. During differentiation, the expression of HSC70 was evaluated by RT-PCR. To determine the function of HSC70 during C2C12 myoblast differentiation, myotube transfection of siR-HSC70 was performed with Lipofectamine 2000 Reagent. Western blot was used to measure the expression of Yin Yang 1 (YY1) after down-regulating HSC70. To further assess if YY1 mediates the pro-differentiation effect of HSC70, a plasmid of YY1 overexpression was used to increase the expression of YY1 in the presence of siR-HSC70-2. The formation of myotubes was visualized by immunofluorescent staining, while the expression levels of MyoD and MyoG were evaluated by RT-PCR.

Results: In this study, we found that HSC70 was up-regulated during C2C12 myoblast differentiation. Knockdown of HSC70 not only inhibited the C2C12 myoblast differentiation but also reduced the expression of MyoD and MyoG. When YY1 protein was over-expressed, it could restore the differentiation in cells with HSC70 knockdown or inhibition.

Conclusions: Collectively, this study demonstrates that HSC70 is involved in the regulation of C2C12 myoblast differentiation via YY1 and may serve as a potential target for a therapeutic strategy to prevent muscle atrophy.
\end{abstract}

Keywords: Heat shock cognate protein 70 (HSC70); myoblast differentiation; Yin Yang 1 (YY1)

Submitted May 26, 2019. Accepted for publication Sep 04, 2019.

doi: $10.21037 /$ atm.2019.09.88

View this article at: http://dx.doi.org/10.21037/atm.2019.09.88

\section{Introduction}

Molecular chaperones are a group of molecules that promote protein folding and help maintain its structure and function. Among them, heat shock proteins (HSPs), can regulate cellular homeostasis to help cells survive in lethal conditions, protecting cells from the injury caused by physical and chemical hazards (1-3).

HSPs can be classified, mainly by their molecular weight, into several families such as the small HSP (sHSP) group, and the HSP40 (4), HSP60 (5), HSP90 (6), HSP110 (7), and HSP100 (8) groups. The members are expressed constitutively or inductively. Among these members, HSP70 has the strongest property of cytoprotection (9). 
Under stress conditions, HSPs will be expressed at a high level, constitutively expressed as Heat shock cognate protein 70 (HSC70), Hsp75, and GRP78 in the endoplasmic reticulum (10). HSC70 is produced as a molecular chaperone protein and constitutes about $1 \%$ of the total protein within a cell. Though mainly produced in the cytoplasm, HSC70 is also found in endosomes, lysosomes, and exosomes (11). Three parts constitute the basic structure of HSC70: an ATP-binding domain, a substrate (peptide) binding domain, and a carboxyl-terminal domain $(2,12)$.

HSC70 has been extensively studied for its role as a clathrin-uncoating ATPase during clathrin-mediated endocytosis (13-16). HSC70 maintains protein homeostasis in both normal and stress conditions, and is thus involved in protein folding, translocation, differentiation, degradation, assembly, and disassembly. It also regulates the process of endocytic and autophagy $(17,18)$. HSC70 protects cells from reactive oxygen species (ROS) produced by mitochondria during inflammatory conditions (19). Furthermore, HSC70 acts as a chaperone for class II major histocompatibility complex proteins, and so any alteration of its trafficking may potentially affect antigenic peptide presentation to the receptor of $\mathrm{T}$ cells, possibly leading to the regulation of a downstream immune response $(20,21)$. HSC70 is reported to be involved in various diseases, and thus has been targeted to become a biomarker for potential diagnosis and therapeutic targets, and consequently developed to be a novel drug for the treatment of these diseases. Much evidence shows that the Hsp70 family plays a vital role in modulating skeletal muscle homeostasis. HSC70 protein expression has been shown to increase during myotube differentiation (22), but how HSC70 functions during skeletal muscle differentiation remains unclear.

In our study, the expression of HSC70 was found to be significantly up-regulated during C2C12 myoblast differentiation. Suppressing HSC70 expression with siRNA not only inhibited the $\mathrm{C} 2 \mathrm{C} 12$ myoblast differentiation but also reduced the expression of MyoD and MyoG. Meanwhile, overexpression of Yin Yang 1 (YY1) protein could promote the myoblast differentiation and restore the differentiation in cells with HSC70 knockdown or inhibition. These findings suggest that HSC70 is critical for C2C12 myoblast differentiation, and could thus be a novel therapeutic target in muscle atrophy treatment.

\section{Methods}

\section{Cell culture and transfection}

C2C12 cells were obtained from ATCC and cultured in Dulbecco's modified Eagle's medium (DMEM) with $10 \%$ fetal bovine serum and $1 \%$ penicillin /streptomycin at $37^{\circ} \mathrm{C}$ with $5 \% \mathrm{CO}_{2}$. During the stage of differentiation, $\mathrm{C} 2 \mathrm{C} 12$ cells at a density of $30,000 / \mathrm{mL}$ was plated on culture plates coated with $0.2 \%$ gelatin. When cell confluence reached $70 \%$, the medium was changed into the differentiation medium (DMEM with $2 \%$ horse serum and $1 \%$ penicillin /streptomycin). Fresh differentiation medium (DMEM with $2 \%$ horse serum and $1 \%$ penicillin /streptomycin) was replaced every $48 \mathrm{~h}$. After 4 days, multinuclear myotubes were formed. All cells were confirmed as mycoplasma-free before use.

C2C12 myotube transfection was performed with Lipofectamine 2000 Reagent (Invitrogen) following the manufacturer's instructions. The siRNA negative control and siR-HSC70 were bought from RiboBio. The procedure for the transfection system was as follows: (I) add $2.5 \mu \mathrm{L}$ SiRNA with a concentration of $100 \mathrm{nM}$ to $100 \mu \mathrm{L}$ of DMEM nutrient medium containing no serum; (II) after mixing well, let stand still for $5 \mathrm{~min}$; (III) add $4 \mu \mathrm{L}$ Lipofectamine 2000 Reagent to $100 \mu \mathrm{L}$ DMEM containing no serum, after mixing well, let stand still for 5 min; (IV) then, add DMEM culture medium containing Lipofectamine 2000 Reagent to the DMEM culture medium containing siRNA by droplet, gently mixing well and letting stand still for $15 \mathrm{~min}$.

The mixture was finally added to $800 \mu \mathrm{L}$ complete medium to prepare the transfection working solution, which was in turn added into $\mathrm{C} 2 \mathrm{C} 12$ cells incubating for $48 \mathrm{~h}$ in DMEM with $10 \%$ fetal bovine serum and $1 \%$ penicillin/ streptomycin. After lipofection, the medium was changed into the differentiation medium (DMEM with $2 \%$ horse serum and $1 \%$ penicillin /streptomycin).

The siRNA target sequences used in this study were as follows: siR-HSC70-1 AGCAUGGAAAGGUGGAAAUUA and siR-HSC70-2 CCGCUUGAGUAA GGAAGAUAU.

The plasmidYY1 CDS sequence obtained from the NCBI was ligated into FUGW plasmid. The PCR primers used in this study were as follows: forward primer: 5'-GC GGGATCCATGGCCTCGGGCGACACC-3'; reverse primer: 5'-GCGGAATTCTCACTGGTTGTTTTTGG 
CTTTA-3'.

\section{Immunofluorescent staining}

C2C12 myotubes were fixed by 4\% PFA for $30 \mathrm{~min}$, permeabilized with $0.5 \%$ Triton $\mathrm{X}-100$ for $10 \mathrm{~min}$, and blocked with $5 \%$ bovine serum albumin (BSA) in PBS for $1 \mathrm{~h}$ at room temperature. C2C12 myotubes were incubated with anti-MHC diluted in $5 \%$ BSA overnight at $4{ }^{\circ} \mathrm{C}$, washed with PBS 3 times, and were then incubated with secondary antibody $\mathrm{Cy} 3$-AffiniPure or FITC rabbit antimouse $\operatorname{IgG}(\mathrm{H}+\mathrm{L})$ at room temperature for $2 \mathrm{~h}$. Images were captured by fluorescence microscope (Leica). The proportion of the number of myotubes containing more than 2 nuclei in all cells reflected the degree of myotube differentiation.

\section{Western blot}

C2C12 myotubes were washed twice with ice-cold PBS and collected in RIPA buffer (Sigma) with phosphatase protease inhibitor. The lysates were centrifuged at 13,000 $\times \mathrm{g}$ at $4{ }^{\circ} \mathrm{C}$ for $30 \mathrm{~min}$. BCA Protein Assay Kit (Beyotime) was used to quantify the protein concentration of the supernatant. Equal amounts of protein samples were separated by $10 \%$ SDS-PAGE gel. Proteins were transferred to a polyvinylidene fluoride membrane. The PVDF membranes were blocked in $5 \%$ non-fat milk in PBST with $0.1 \%$ Tween 20 for $1 \mathrm{~h}$ at room temperature and then incubated with primary antibodies diluted in 5\% BSA-TPBS overnight at $4{ }^{\circ} \mathrm{C}$. The following primary antibodies were used: YY1 (1:1,000, Proteintech) and GAPDH-HRP (1:5,000, Santa). The PVDF membranes were washed with PBS 3 times. Secondary antibodies including anti-mouse (1:5,000, Multi Sciences) and anti-rabbit (1:5,000, Multi Sciences) IgG horseradish peroxidase-conjugated secondary antibody, were incubated with membranes for $2 \mathrm{~h}$ in $5 \%$ non-fat milk in TPBS. ECL Chemiluminescent Kit (Thermo Fisher) was used to visualize the antibody-antigen interaction. Finally, chemical luminescence of membranes was detected using a BioRad luminescent imaging system.

\section{Quantitative real-time polymerase chain reactions}

Total RNA extraction from C2C12 myotubes was performed by RNeasy Mini Kit (Qiagen), according to the manufacturer's instructions. After the concentration was measured, the same amount of RNA was taken for reverse transcription. cDNA was synthesized by using Takara PrimeScript 1st Strand cDNA Synthesis Kit and was subjected to the quantitative PCR with Takara SYBR Premix Ex TaqTM. The reaction procedure was set as followings: $95^{\circ} \mathrm{C}, 3 \mathrm{~min} ; 95^{\circ} \mathrm{C}, 15 \mathrm{~s} ; 60{ }^{\circ} \mathrm{C}, 30 \mathrm{~s} ; 72^{\circ} \mathrm{C}, 30 \mathrm{~s} ; 40$ cycles. $18 \mathrm{~S}$ rRNA was used as an internal control. The relative changes in the expression of the gene were calculated using the $2^{-\Delta \Delta \mathrm{Ct}}$ method. The primer sequences were as follows: mus-18S F: TCAAGAACGAAAGTCGGAGG, R: GGACATC TAA G G G CATCAC; mus H S C 70 F: TCTCGGCACCACCTACTCC, R: CTACGCCCGATCAGACGTTT; musMyoD F: CCACTCCGGGACATAGACTTG, R: AAAAGCGCAGGTCTGGTGAG; musMyoG F: GAGACATCCCCCTATTTCTACCA, R: GCTCAGTCCGCTCATAGCC; mus-YY1 F : CAGTGGTTGAAGA G CA GATCAT, R : AGGGAGTTTCTTGCCTGTCAT.

\section{Statistical analysis}

Data are expressed as mean \pm SEM. Two-tailed Student's $t$-test was applied for comparisons between the value of the two groups. One-way ANOVA test was performed to compare multiple groups after Bonferroni's post hoc test. All analyses were performed using GraphPad Prism 6.0. Differences were considered when criterion significance with $\mathrm{P}<0.05$ ( ${ }^{*}, \mathrm{P}<0.05,{ }^{* *}, \mathrm{P}<0.01$ ).

\section{Results}

\section{HSC70 is up-regulated during C2C12 myoblast differentiation}

To determine the expression changes during myoblast differentiation, we analyzed its level of expression in myotubes differentiated from C2C12 (Figure 1A). The PCR result showed that HSC70 was up-regulated when multinuclear myotubes were formed on the fourth day (Figure 1B).

\section{HSC70 is critical for C2C12 myoblast differentiation}

It has been found that HSC70 is up-regulated during C2C12 myoblast differentiation. To determine the function of HSC70 during C2C12 myoblast differentiation, the expression of HSC70 was inhibited with siRNA. C2C12 cells were transfected with siR-HSC70-1 and siR-HSC70-2 

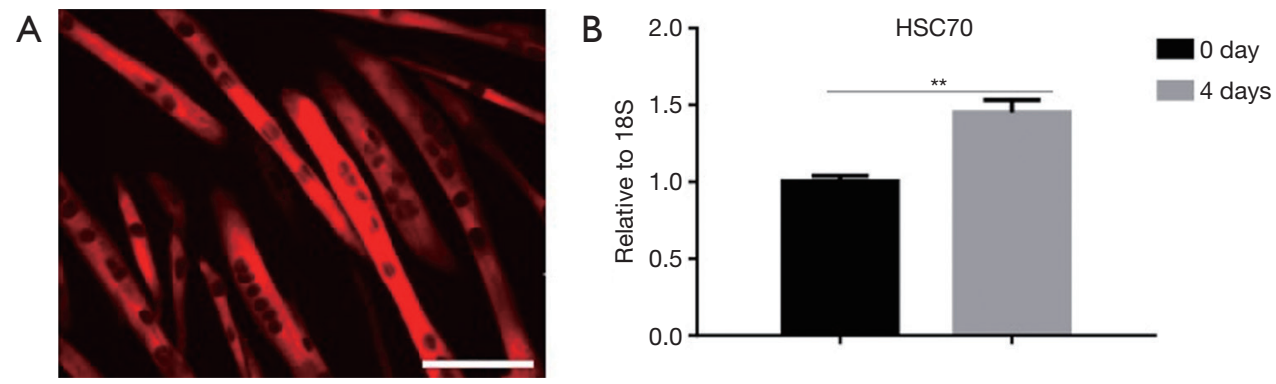

Figure 1 HSC70 is up-regulated during C2C12 myoblast differentiation. (A) Immunofluorescent staining with MyHC cy3 stained (red) for C2C12 myotubes differentiated from primary myoblasts (scale bar, $100 \mu \mathrm{m}$ ); (B) the qRT-PCR analysis of HSC70 showed the expression of HSC70 increased markedly ( $\mathrm{n}=4$ per group). Data presented are the mean $\pm \mathrm{SEM}$ of the three independent experiments. ${ }^{* *}, \mathrm{P}<0.01$.

and incubated $48 \mathrm{~h}$ before $\mathrm{C} 2 \mathrm{C} 12$ myoblast differentiation (Figure $2 A$ ). Immunofluorescent staining was performed to assess the effect of HSC70 on C2C12 differentiation. Myotube formation was suppressed in $\mathrm{C} 2 \mathrm{C} 12$ cells transfected with siR-HSC70-1 and siR-HSC70-2 (Figure 2B). Also, mRNA expressions of MyoD and MyoG were significantly reduced (Figure 2C). These results suggest that HSC70 knockdown inhibits C2C12 differentiation.

\section{HSC70 positively regulates the expression of YY1 protein}

We measured the protein abundance of YY1 after downregulating $\mathrm{HSC} 70$ and found that the expression of YY1 decreased with the down-regulation of HSC70 at 4 days (Figure $3 A$ ). Also, we found the YYI protein expression was increased during C2C12 myoblast differentiation (Figure 3B).

\section{YY1 promotes myoblast differentiation}

To further assess if YY1 mediates the pro-differentiation effect of HSC70, plasmid of YY1-overexpression was used to increase the expression of $\mathrm{YY} 1$ in the presence of siRHSC70-2 (Figure 4A). We found that YY1 overexpression could accelerate myoblast differentiation. Moreover, it could also restore the inhibition effect of siR-HSC70-2 on myoblast differentiation, contributing to myotube formation and increasing the mRNA expressions of MyoD and MyoG (Figure 4B,C). These results show that YY1 expression promotes myoblast differentiation.

\section{Discussion}

HSC70, as a molecular chaperone, regulates various cellular functions by interacting with many other molecules. It is found to be involved in various diseases and may become a potential biomarker for diagnosis and therapeutic targets for design, discovery, and development of novel drugs (23).

Now, more studies have shown that HSC70 plays a vital role in the physiological activities of muscles. Overexpression of Hsp70 is capable of inhibiting key atrophy signaling pathways and thus prevents skeletal muscle atrophy (24). Hsp70 knockout consistently results in sustained inflammation and fibrosis in skeletal muscles after cardiotoxin injection (25). However, only a few studies focus on the role of HSC70 in the regulation of myoblast differentiation. In this study, we found that the expression of HSC70 is significantly up-regulated during C2C12 myoblast differentiation. Suppression of HSC70 with siRNA not only inhibited the C2C12 myoblast differentiation but also reduced the expression of $\mathrm{MyoD}$ and MyoG, two proteins mainly involved in the regulation of myoblast differentiation $(26,27)$. This evidence suggests that HSC70 is critical for $\mathrm{C} 2 \mathrm{C} 12$ myoblast differentiation.

YY1 participates in the lineage differentiation of skeletal cells, and also in several disease pathways such as dystrophic muscle disease (28-30). It is reported that YY1 inhibits vascular smooth muscle cell growth (31). The expression of YY1 has been shown to gradually decreased during C2C12 myoblast differentiation (32), while miRNA-34c has been observed inhibiting myoblast proliferation by targeting YY1 (33). The regulation of miR-1 by YY1 holds functional importance for both C2C12 myogenic differentiation and injury-induced muscle regeneration (34). LncRNA (Yam-1), whose expression YY1 could positively regulate, is downregulated upon differentiation and acts as an inhibitor of myogenesis (29). Lnc-YY1 functions to promote myogenesis by interacting with YY1 (35). Also, YY1 triggers the up-regulation of miR-29b, which contributes 

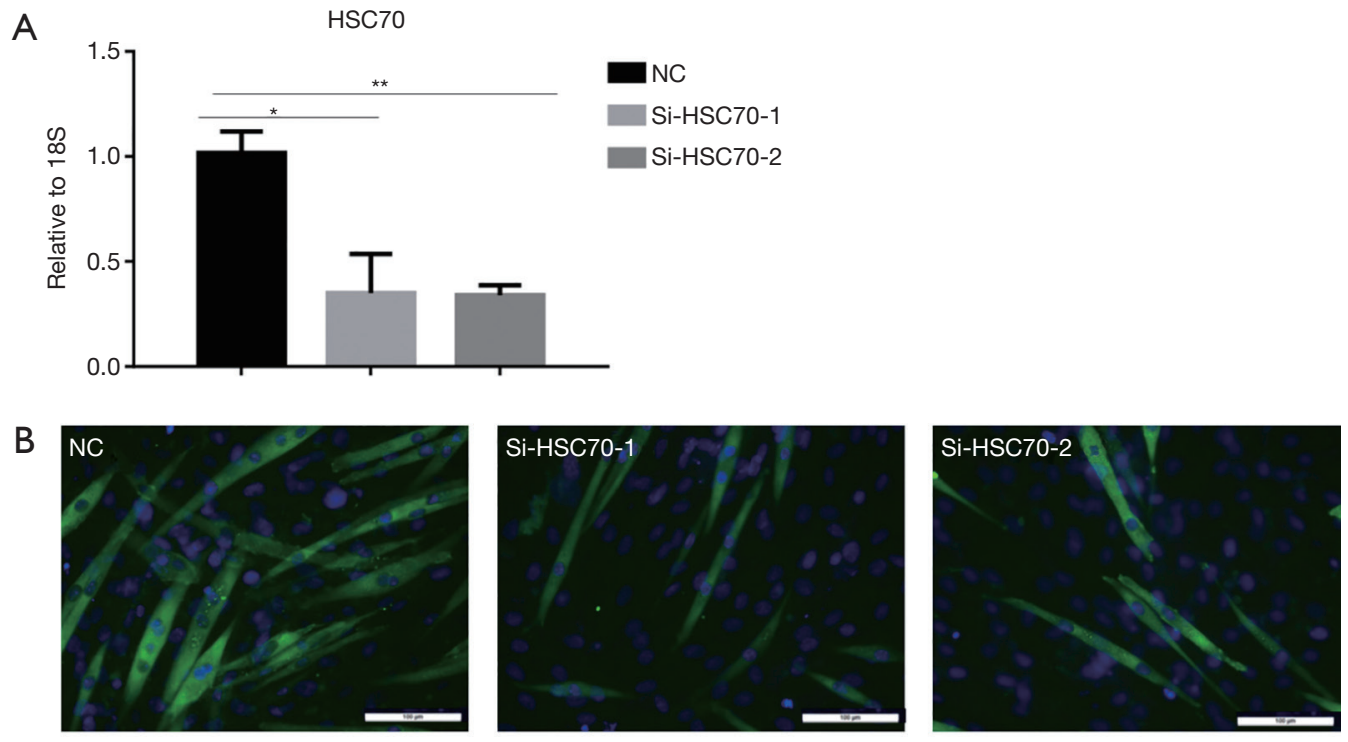

C

MyoD

MyoG
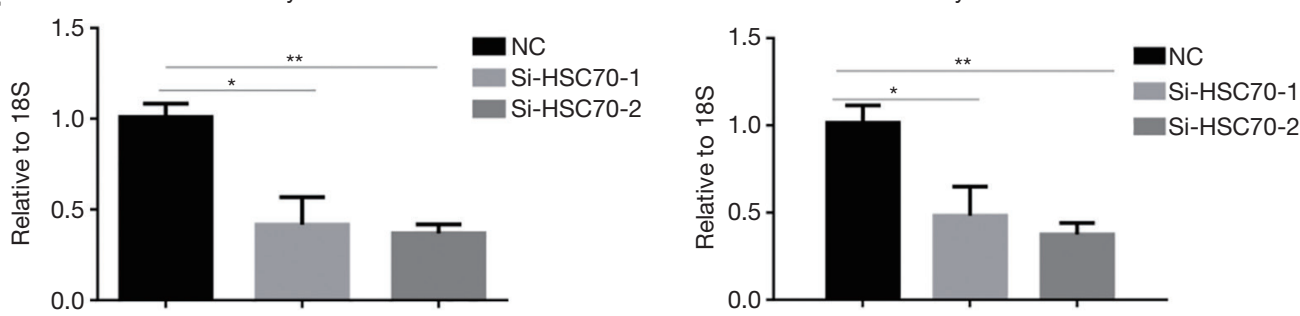

Figure 2 HSC70 is critical for C2C12 myoblast differentiation. (A) qRT-PCR analysis showed reduced HSC70 in C2C12 myotubes treated with siRNA compared to NC control (n=4); (B) immunofluorescent staining with MyHC FTIC stained (green) for C2C12 myotubes transfected with siR-HSC70 (scale bar, $100 \mu \mathrm{m}$ ); (C) qRT-PCR analysis showed downregulated MyoD and MyoG expressions in C2C12 myotubes transfected with siR-HSC70 ( $\mathrm{n}=4$ per group). Data presented are the mean \pm SEM of three independent experiments. * $\mathrm{P}<0.05$, **, $\mathrm{P}<0.01$.

A
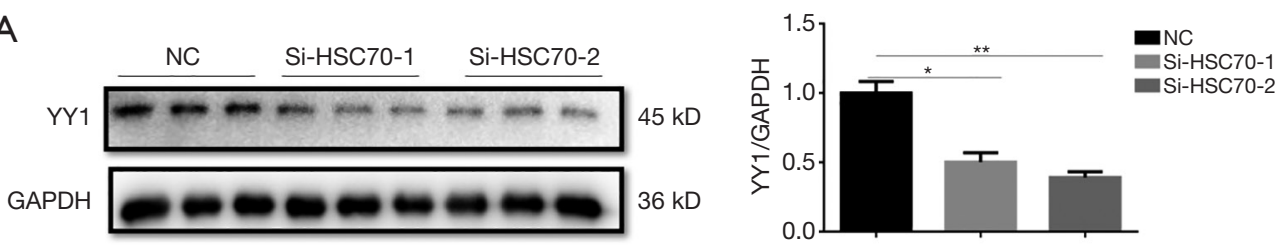

B

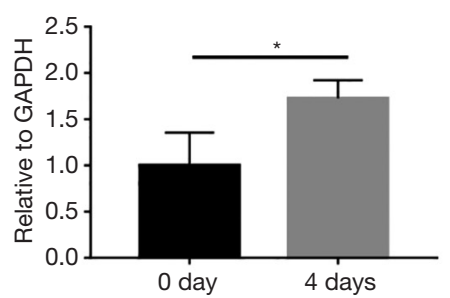

Figure 3 HSC70 positively regulates the expression of YY1 protein. (A) Western blot analysis showed decreased YY1 protein level in C2C12 myotubes transfected with siR-HSC70 on day 4 (n=3); (B) Western blot analysis showed increased YY1 protein level during C2C12 myotube differentiation $(\mathrm{n}=3)$. Data presented are the mean \pm SEM of three independent experiments. * $\mathrm{P}<0.05,{ }^{* *}, \mathrm{P}<0.01$. 
A

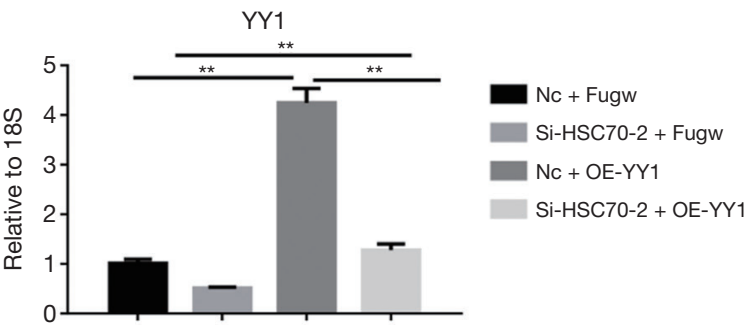

B
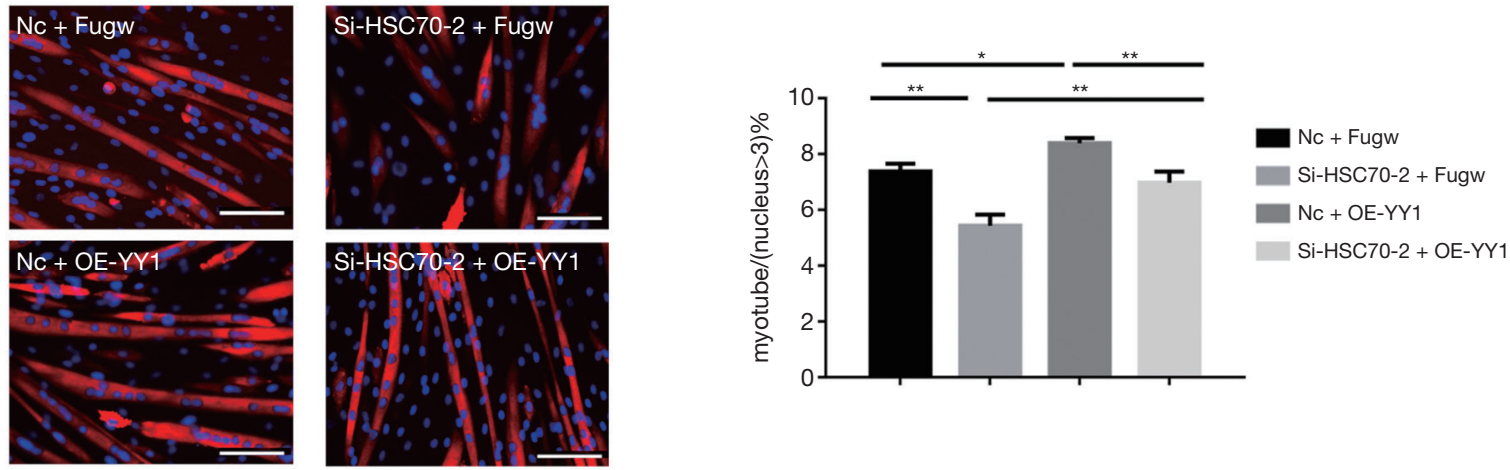

C
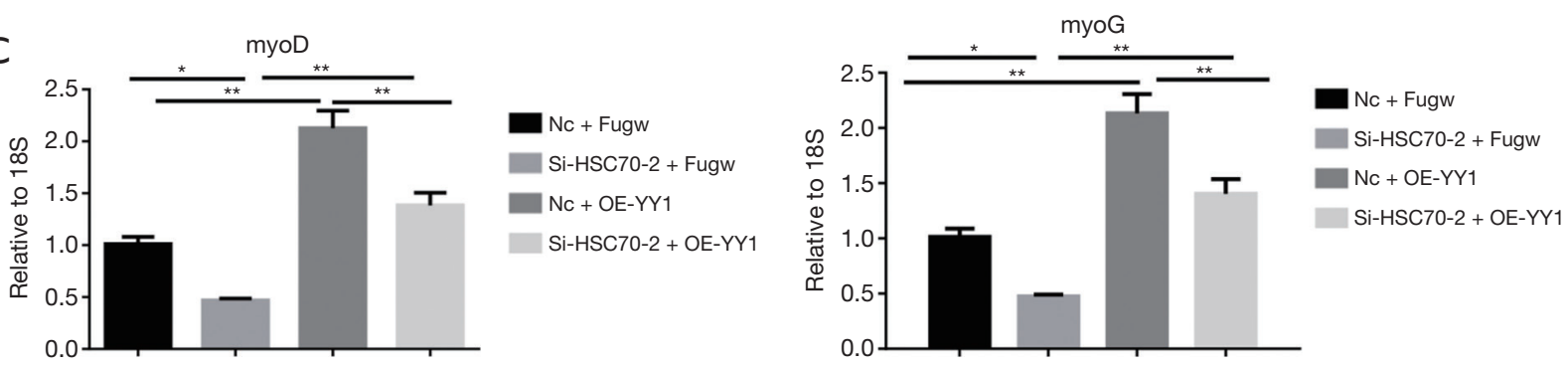

Figure 4 YY1 promotes myoblast differentiation. (A) qRT-PCR analysis showing that overexpression of YY1 in C2C12 myotubes transfected with Fugw-YY1 or siR-HSC70 (n=6); (B) immunofluorescent staining for C2C12 myotubes transfected with Fugw-YY1 or siRHSC70 (scale bar, $100 \mu \mathrm{m}$ ); (C) qRT-PCR analysis showing that overexpression of YY1 increased siR-HSC70-induced downregulation of MyoD and MyoG in C2C12 myotubes ( $\mathrm{n}=6$ per group). Data presented are the mean $\pm \mathrm{SEM}$ of three independent experiments. * $\mathrm{P}<0.05$, **, $\mathrm{P}<0.01$.

to multiple types of muscle atrophy (36). In this study, the overexpression of YY1 protein attenuated the inhibition of myoblast differentiation induced by HSC70 knockdown. YY1 protein expression was increased during skeletal muscle regeneration, while muscle-specific deletion of YY1 led to severe deformity of diaphragm muscle formation (37). To our knowledge, the cooperation of co-chaperones enables HSC70 to fully perform its function, so the specific mechanism of HSC70 and YY1-induced myoblast differentiation should be further explored, and hopefully, identified in the future.

\section{Conclusions}

In conclusion, our study shows that HSC70 is critical for C2C12 myoblast differentiation, a process also regulated by YY1 protein. This finding suggests that HSC70 might become a novel therapeutic target of muscle-related disease.

\section{Acknowledgments}

Funding: This work was supported by grants from the National Natural Science Foundation of China 
(ID:81701218 to Lei Chen, ID:81873774 to Yan Yu), the Sailing Program from the Shanghai Science and Technology Commission (ID:16YF1410300 to Lei Chen), the Tongji Hospital of Shanghai (ID: HBRC1607 to Lei Chen, KPB1608 to Yeqing Sun), and Guizhou Provincial People's Hospital Doctor's Fund [ID: GZSYBS(2017)12 to Tao Guo].

\section{Footnote}

Conflicts of Interest: The authors have no conflicts of interest to declare.

Ethical Statement: The authors are accountable for all aspects of the work in ensuring that questions related to the accuracy or integrity of any part of the work are appropriately investigated and resolved. This article does not involve any patients or animals, and the ethical review is exempted.

\section{References}

1. Smith DF, Whitesell L, Katsanis E. Molecular chaperones: biology and prospects for pharmacological intervention. Pharmacol Rev 1998;50:493-514.

2. Tsukahara F, Yoshioka T, Muraki T. Molecular and functional characterization of HSC54, a novel variant of human heat-shock cognate protein 70. Mol Pharmacol 2000;58:1257-63.

3. Rodriguez-Ariza A, Lopez-Sanchez LM, Gonzalez R, et al. Altered protein expression and protein nitration pattern during d-galactosamine-induced cell death in human hepatocytes: a proteomic analysis. Liver Int 2005;25:1259-69.

4. Alderson TR, Kim JH, Markley JL. Dynamical Structures of Hsp70 and Hsp70-Hsp40 Complexes. Structure 2016;24:1014-30.

5. Wiechmann K, Muller H, Konig S, et al. Mitochondrial Chaperonin HSP60 Is the Apoptosis-Related Target for Myrtucommulone. Cell Chem Biol 2017;24:614-23.e6.

6. Krishna P, Gloor G. The Hsp90 family of proteins in Arabidopsis thaliana. Cell Stress Chaperones 2001;6:238-46.

7. Zininga T, Achilonu I, Hoppe H, et al. Plasmodium falciparum Hsp70-z, an Hsp110 homologue, exhibits independent chaperone activity and interacts with Hsp70-1 in a nucleotide-dependent fashion. Cell Stress Chaperones 2016;21:499-513.
8. Pulido P, Llamas E, Llorente B, et al. Specific Hsp100 Chaperones Determine the Fate of the First Enzyme of the Plastidial Isoprenoid Pathway for Either Refolding or Degradation by the Stromal Clp Protease in Arabidopsis. PLoS Genet 2016;12:e1005824.

9. Carmody S, Wu XL, Lin H, et al. Cytoprotection by electromagnetic field-induced hsp70: a model for clinical application. J Cell Biochem 2000;79:453-9.

10. Li P, Wang J, Zou Y, et al. Interaction of Hsp90AA1 with phospholipids stabilizes membranes under stress conditions. Biochim Biophys Acta Biomembr 2019;1861:457-65.

11. Stricher F, Macri C, Ruff M, et al. HSPA8/HSC70 chaperone protein: structure, function, and chemical targeting. Autophagy 2013;9:1937-54.

12. Sullivan CS, Pipas JM. T antigens of simian virus 40: molecular chaperones for viral replication and tumorigenesis. Microbiol Mol Biol Rev 2002;66:179-202.

13. Braell WA, Schlossman DM, Schmid SL, et al. Dissociation of clathrin coats coupled to the hydrolysis of ATP: role of an uncoating ATPase. J Cell Biol 1984;99:734-41.

14. Schlossman DM, Schmid SL, Braell WA, et al. An enzyme that removes clathrin coats: purification of an uncoating ATPase. J Cell Biol 1984;99:723-33.

15. Ungewickell E. The 70-kd mammalian heat shock proteins are structurally and functionally related to the uncoating protein that releases clathrin triskelia from coated vesicles. EMBO J 1985;4:3385-91.

16. Chappell TG, Welch WJ, Schlossman DM, et al. Uncoating ATPase is a member of the 70 kilodalton family of stress proteins. Cell 1986;45:3-13.

17. Deffit SN, Blum JS. A central role for HSC70 in regulating antigen trafficking and MHC class II presentation. Mol Immunol 2015;68:85-8.

18. Tian Y, Xu H, Farooq AA, et al. Maslinic acid induces autophagy by down-regulating HSPA8 in pancreatic cancer cells. Phytother Res 2018;32:1320-31.

19. Wang F, Bonam SR, Schall N, et al. Blocking nuclear export of HSPA8 after heat shock stress severely alters cell survival. Sci Rep 2018;8:16820.

20. Srivastava P. Roles of heat-shock proteins in innate and adaptive immunity. Nat Rev Immunol 2002;2:185-94.

21. Deffit SN, Blum JS. Macronutrient deprivation modulates antigen trafficking and immune recognition through HSC70 accessibility. J Immunol 2015;194:1446-53.

22. Fan W, Gao XK, Rao XS, et al. Hsp70 Interacts with Mitogen-Activated Protein Kinase (MAPK)-Activated 
Protein Kinase 2 To Regulate p38MAPK Stability and Myoblast Differentiation during Skeletal Muscle Regeneration. Mol Cell Biol 2018. doi: 10.1128/ MCB.00211-18.

23. Liu T, Daniels CK, Cao S. Comprehensive review on the HSC70 functions, interactions with related molecules and involvement in clinical diseases and therapeutic potential. Pharmacol Ther 2012;136:354-74.

24. Senf SM, Dodd SL, McClung JM, et al. Hsp70 overexpression inhibits NF-kappaB and Foxo3a transcriptional activities and prevents skeletal muscle atrophy. FASEB J 2008;22:3836-45.

25. Senf SM, Howard TM, Ahn B, et al. Loss of the inducible Hsp70 delays the inflammatory response to skeletal muscle injury and severely impairs muscle regeneration. PLoS One 2013;8:e62687.

26. Xu D, Wang L, Jiang Z, et al. A new hypoglycemic mechanism of catalpol revealed by enhancing MyoD/ MyoG-mediated myogenesis. Life Sci 2018;209:313-23.

27. Huang Y, Lai X, Hu L, et al. Over-expression of DEC1 inhibits myogenic differentiation by modulating MyoG activity in bovine satellite cell. J Cell Physiol 2018;233:9365-74.

28. Lee TC, Zhang Y, Schwartz RJ. Bifunctional transcriptional properties of $\mathrm{YY} 1$ in regulating muscle actin and c-myc gene expression during myogenesis. Oncogene 1994;9:1047-52.

29. Lu L, Sun K, Chen X, et al. Genome-wide survey by ChIP-seq reveals YY1 regulation of lincRNAs in skeletal myogenesis. EMBO J 2013;32:2575-88.

30. Gabellini D, Green MR, Tupler R. Inappropriate

Cite this article as: Chen L, Guo T, Yu Y, Sun Y, Yu G, Cheng L. Heat shock cognate protein 70 promotes the differentiation of C2C12 myoblast and targets Yin Yang 1. Ann Transl Med 2019;7(20):551. doi: 10.21037/atm.2019.09.88 gene activation in FSHD: a repressor complex binds a chromosomal repeat deleted in dystrophic muscle. Cell 2002;110:339-48.

31. Santiago FS, Ishii H, Shafi S, et al. Yin Yang-1 inhibits vascular smooth muscle cell growth and intimal thickening by repressing p21WAF1/Cip1 transcription and p21WAF1/Cip1-Cdk4-cyclin D1 assembly. Circ Res 2007;101:146-55.

32. Zhou L, Wang L, Lu L, et al. A novel target of microRNA-29, Ring1 and YY1-binding protein (Rybp), negatively regulates skeletal myogenesis. J Biol Chem 2012;287:25255-65.

33. Wang M, Liu C, Su Y, et al. miRNA-34c inhibits myoblasts proliferation by targeting YY1. Cell Cycle 2017;16:1661-72.

34. Lu L, Zhou L, Chen EZ, et al. A Novel YY1-miR-1 regulatory circuit in skeletal myogenesis revealed by genome-wide prediction of YY1-miRNA network. PLoS One 2012;7:e27596.

35. Sun K, Zhou L, Zhao Y, et al. Genome-wide RNA-seq and ChIP-seq reveal Linc-YY1 function in regulating YY1/ PRC2 activity during skeletal myogenesis. Genom Data 2016;7:247-9.

36. Li J, Chan MC, Yu Y, et al. miR-29b contributes to multiple types of muscle atrophy. Nat Commun 2017;8:15201.

37. Chen F, Zhou J, Li Y, et al. YY1 regulates skeletal muscle regeneration through controlling metabolic reprogramming of satellite cells. EMBO J 2019. doi: 10.15252/embj.201899727. 